\title{
A parallel finite-difference approach for three-dimensional transient electromagnetic modeling with galvanic sources
}

\author{
Michael Commer \\ Institute of Geophysics und Meteorology, University of Cologne, Germany \\ Gregory Newman*
}

Earth Science Division, Lawrence Berkeley National Laboratory, One Cyclotron

Road, MS 90-1116

(February 9, 2004)

\begin{abstract}
A parallel finite-difference algorithm for the solution of diffusive, threedimensional (3-D) transient electromagnetic field simulations is presented. The purpose of the scheme is the simulation of both electric fields and time derivative of magnetic fields generated by galvanic sources (grounded wires) over arbitrarily complicated distributions of conductivity and magnetic permeability. Using a staggered grid and a modified DuFort-Frankel method, the scheme steps Maxwell's equations in time. Electric field initialization is done by a conjugate-gradient solution of a 3-D Poisson problem, as is common in 3-D resistivity modeling. Instead of calculating the initial magnetic field directly, its time derivative and curl are employed in order to advance the electric field in time. A divergence-free condition is enforced for both the magnetic field time derivative and the total conduction current density, providing accurate results at late times. In order to simulate large realistic earth models, the
\end{abstract}

\footnotetext{
*formerly at Sandia National Laboratories, PO Box 5800, Albuquerque, NM 87185-0750
} 
algorithm has been designed to run on parallel computer platforms. The upward continuation boundary condition for a stable solution in the infinitely resistive air layer involves a two-dimensional parallel FFT. Example simulations are compared with analytical, integral-equation and spectral Lanczos decomposition solutions and demonstrate the accuracy of the scheme.

\section{INTRODUCTION}

Solving 3-D transient electromagnetic (TEM) problems is important in understanding the effects of multidimensional conductivity structures. As data quality and quantity arising from TEM surveys have tended to increase together with computational capabilities, routine interpretation is becoming more and more multidimensional in character. This is also preferable in order to enable multidisciplinary interpretation approaches as a means to achieve an earth model with minimum ambiguity.

TEM methods provide very useful additional information for exploration problems associated with oil exploration, geological hazards and hydrological investigations (Strack, 1992). Shallow exploration typically involves TEM systems that employ loops as transmitting antennas with an inductive coupling to the Earth. Such systems can be deployed rapidly and more easily than grounded wires. The grounded wire is a more complex source. However, it is often used in deep soundings because the field falls off less rapidly at large distances and generation of adequate field levels is difficult with loop sources (Spies and Frischknecht, 1991). The Long-Offset TEM (LOTEM) technique typically uses a long grounded wire for deep crustal studies (Hördt et al., 1992; Thern et al., 1996; Hördt et al., 2000b; Mitsuhata et al., 2002), but additional complexities arise with such field sourcing. The typical transmitter lengths and receiver distances often do not allow the grounded wire to be approximated by a mathematical dipole or infinite line source, and the galvanic nature of the source 
must be considered in the interpretation of the data.

Finite-difference (FD) methods have been used extensively due to their simplicity in solving 2-D and 3-D time domain modeling problems. Goldman and Stoyer (1983) addressed the modeling of transients in a simplified axially symmetric media with an implicit and 2-D formulation. A simulation of TEM surveys over thin 2-D vertical conductors embedded in a half-space with overburden is described by Oristaglio and Hohmann (1984). Their numerical method for solving the 2-D transverse electric mode of Maxwell's equations is based on an explicit FD scheme originally proposed by DuFort and Frankel (1953) and generalized to inhomogeneous models and grids with an irregular spacing. For 3-D field simulation, Druskin and Knizhnerman (1988) developed a spectral differential-difference technique, where the differential equations are solved using a FD staggered-grid scheme invented earlier by Yee (1966). This scheme preserves the flux conditions on current density and magnetic induction fields, which is critical in 3-D field simulation. Wang and Hohmann (1993) presented an explicit 3-D FD scheme for loop sources that also employs a staggered grid. Their scheme uses a modification of the DuFort-Frankel method and incorporates analytical impulse response solutions for an assumed homogeneous earth model as initial conditions.

In this paper we present a FD scheme for the simulation of fields generated by galvanic sources. Although the basis of our method is the FD scheme presented by Wang and Hohmann (1993), our scheme differs from their solution in some important key aspects. First, the initial conditions are computed for an arbitrarily geological media. This involves the solution of a 3-D Poisson problem prior to the time-stepping process in order to treat the presence of a static DC electric field caused by the galvanic source. We are thus able to effectively simulate the step response of the electric field, because the Earth is a lossy medium. At the earliest times, however, this is not the case because our discrete model cannot support the high frequency content of the step waveform. Nevertheless, the high frequency content damps out 
rapidly with time, and is not needed for accurate field simulation over the time range of interest. Due to the fact that only electric fields and the time derivative of the magnetic induction are typically measured with grounded-wire TEM systems, we wish to avoid the additional computation of a magnetometric resistivity (MMR) problem (Edwards and Nabighian, 1991; Haber, 2000). Otherwise, this would be required for the calculation of the initial static magnetic field. It will be outlined how we work only with the time derivative of the magnetic induction and the curl of the magnetic field in order to advance the electric field. Second, we enforce a divergence-free condition on both the magnetic induction time derivative and the total conduction current density to ensure accurate results at late times. At last, to address the inherently large computational effort of explicit time-stepping solutions, the algorithm has been designed to run on distributed computer platforms. This involves a message passing scheme that is different from the FD scheme for frequency-domain forward modeling (Alumbaugh et al., 1996; Newman and Alumbaugh, 1997) and will be discussed at length in the parallel implementation section of the paper.

\section{METHOD}

In order to simulate the propagation of the total electric field intensity $\mathbf{e}=\mathbf{e}(\mathbf{r}, t)$, the magnetic field $\mathbf{h}=\mathbf{h}(\mathbf{r}, t)$ and the magnetic induction $\mathbf{b}=\mathbf{b}(\mathbf{r}, t)$ as a function of the position vector $\mathbf{r}=(x, y, z)$ and time $t$, we review Maxwell's equations in the time domain:

$$
\begin{array}{r}
\nabla \times \mathbf{e}+\frac{\partial \mathbf{b}}{\partial t}=0 \\
\nabla \times \mathbf{h}-\sigma \mathbf{e}-\epsilon \frac{\partial \mathbf{e}}{\partial t}=\mathbf{j}_{s}
\end{array}
$$

where $\sigma, \mu$ and $\epsilon$ are the conductivity, magnetic permeability and permittivity of the 3-D heterogeneous media, respectively. The primary electric current density $\mathbf{j}_{s}$ is impressed by the transmitting source. It is quantified by the electric current per unit 
volume, $I$, integrated along the extent $L$ of the line source,

$$
\mathbf{j}_{s}=\int_{L} I[1-H(t)] d l
$$

where $H(t)$ is the Heaviside step function. Signal generation takes place by shutting off the initially steady current through the line source of finite length.

\section{Method of discretization in time}

Following Wang and Hohmann (1993), we use a scheme that employs a staggeredgrid (Yee, 1966) in conjunction with a modified version of the DuFort-Frankel method (DuFort and Frankel, 1953; Oristaglio and Hohmann, 1984) for time-stepping equations (1). The DuFort-Frankel method is explicit, because each equation of the equation system gives one of the field unknowns $u^{n+1}$ at a time $(n+1) \Delta t$ directly in terms of known quantities $u^{n}$ from an earlier time step $n \Delta t$ (Richtmyer and Morton, 1967). The set of equations (1) represents wave phenomena, while over longer times the field obtains a diffusive nature due to the domination of low-frequencies. Therefore, the equations are very stiff in time (Ascher and Petzold, 1998) and thus require very small time steps $\Delta t$ to retain stability with an explicit method (Haber et al., 2002). Implicit methods are more suitable if displacement currents are negligible, because such schemes provide accurate results for larger time steps. In contrast to explicit schemes,

a set of simultaneous linear equations is solved to obtain $u^{n+1}$, which requires matrix inversions at each time step. Examples for implicit methods are Crank-Nicolson or the backward-difference method (Lapidus and Pinder, 1982; Goldman and Stoyer, 1983; Ascher and Petzold, 1998).

It would be beyond the scope of this paper to exemplify a comparison of explicit versus implicit methods in terms of computational efficiency. We are aware that the inherently high computational effort of the explicit scheme remains a major drawback, but this can be mitigated using the modified DuFort-Frankel method. This method is 
also easy to implement and stable and one does not have to deal with problems arising from the inversion of large linear systems. An explicit scheme is also extremely reliable for producing accurate results over a large dynamic time range $\left(10^{-7}-1 \mathrm{sec}\right)$, necessary for inverse modeling problems (Commer, 2003; Newman and Commer, 2004).

\section{Method of discretization in space}

An earth model is represented by a 3-D Cartesian mesh of rectangular cells. Complicated underground structures can then be modeled by assigning different electrical and magnetic properties to the grid cells. The staggered grid provides an accurate scheme for the discretization of the above equations on the 3-D mesh. As shown in Figure 1, electric fields are sampled on cell edges and magnetic fields on the center of cell faces. This is useful for the discretization of the curl operations. Elementary electric loops are formed by the four components surrounding a face of a grid cell (Figure 1a), and are required for the calculation of the corresponding magnetic field with normal orientation. Each electric field component in turn is surrounded by an elementary loop of four magnetic components as shown by Figure $1 \mathrm{~b}$ and exemplified by the dashed rectangle around $e_{x}$. Each electric field cartesian component is assigned to a directional conductivity that is evaluated by averaging the conductivities of the four prisms on a particular cell edge. A weighting function evaluated as the ratio of the area of a particular prism cut by the magnetic loop to the total area of the loop is employed (Wang and Hohmann, 1993). Similarly, the magnetic permeability is averaged geometrically across the cell faces to correspond to the location of the magnetic fields (Alumbaugh et al., 1996).

Note that typical grounded-wire TEM equipment uses receiver loops for measuring the time derivative of the magnetic field. Instead of magnetic fields, we thus focus on

the simulation of magnetic induction time derivative measurements $\dot{\mathbf{b}}$ in addition to electric fields. This allows to avoid the numerical and rather elaborate calculation of 
the static magnetic field prior to source shutoff, which would involve a MMR problem. Stepping forward in time is thus also based on $\mathbf{b}$ instead of $\mathbf{h}$. Our scheme involves calculating the curl of the magnetic field directly from $\dot{\mathbf{b}}$ in order to advance the electric field, as will be further clarified by the description of the updating scheme. Note, that on the staggered grid the components of $\dot{\mathbf{b}}$ are sampled like magnetic fields.

\section{Stability considerations}

For the treatment of TEM fields, the diffusive approximation of equations (1), i.e. without displacement currents, needs to be considered. However, the DuFortFrankel method is actually realized by introducing a hyperbolic displacement term $\tilde{\epsilon} \frac{\partial \mathbf{e}}{\partial t}$ to the diffusion equation, which is also referred to as a modified DuFort-Frankel version by Wang and Hohmann (1993). Hence, the displacement term in equation (1b) now represents an artificial add-on with a fictitious permittivity $\tilde{\epsilon}$ and we solve a system which is consistent with the damped wave form. It can be shown that with the DuFort-Frankel method this system is unconditionally stable, provided the CourantFriedrichs-Levy condition is satisfied (Oristaglio and Hohmann, 1984; Adhidjaja and Hohmann, 1989),

$$
\frac{1}{\sqrt{\mu \tilde{\epsilon}}} \leq \frac{1}{\sqrt{3}} \frac{\Delta}{\Delta t_{n}}
$$

where $\Delta$ represents the grid spacing and $\Delta t_{n}$ the variable time step. The artificial permittivity can be orders of magnitude larger than that observed for nonpolarizable earth materials, thereby allowing for a much coarser time sampling of the fields. From equation (2), one obtains

$$
\tilde{\epsilon} \geq \frac{3}{\mu_{\min }}\left(\frac{\Delta t_{n}}{\Delta_{\min }}\right)^{2}
$$

where $\Delta_{\text {min }}$ is the smallest FD grid spacing and $\mu_{\text {min }}$ is the minimum magnetic permeability of a given earth model. Nevertheless, equation (3) indicates, that the 
time step has to be bounded in order to avoid a dominating displacement current. Seen from a different angle, Richtmyer and Morton (1967) showed that the DuFortFrankel method applied to the discretization of the diffusion equation results in a truncation error of order $\frac{\Delta t^{2}}{\Delta^{2}}$. Given a fixed grid size $\Delta$, this requires a small time step in order to retain consistency with the diffusion equation. In practice we choose the initial time step $\Delta t_{0}$ such that the domination of diffusion after the earliest time $t$ of the simulated measurements is valid (Oristaglio and Hohmann, 1984; Adhidjaja and Hohmann, 1989),

$$
\Delta t_{0} \ll \sqrt{\frac{\mu_{\min } \sigma_{\min } t}{6}} \Delta_{\min }
$$

where $\sigma_{\min }$ is the minimum value of an arbitrary conductivity structure.

Another important issue for stability is the explicit enforcement of a vanishing divergence for both the time derivative of the magnetic induction and the total conduction current density

$$
\begin{aligned}
& \nabla \cdot \dot{\mathbf{b}}=0 \\
& \nabla \cdot \mathbf{j}=0
\end{aligned}
$$

As will be further clarified by the description of the updating scheme, the avoidance of an explicit calculation of magnetic fields requires that both these divergence-free conditions be enforced in order to get stable results at late times. Outlined in more detail by Wang and Hohmann (1993), the enforcement avoids distorting arbitrary gradient fields that can creep into the EM field simulation at later times.

\section{Boundary conditions}

The staggered grid allows to enlarge the spacings of the grid nodes with increasing offset from the source position. We thus simply extend the subsurface boundaries sufficiently far from the source such that both the tangential electric and normal mag- 
netic induction time derivative components satisfy a homogeneous Dirichlet boundary condition. The distance of the mesh boundaries from the source may have to be adapted to the underlying earth model. If the boundaries are too close to transmitter or receiver positions, we observe erroneous results due to grid reflection at late times. This problem becomes more severe for highly-resistive backgrounds, where the diffusing fields face less attenuation than in a more conductive earth.

An important feature for electromagnetic modeling is the treatment of the earthair interface by an upward-continuation boundary condition, which is used to avoid having to include the air layer in the FD grid (Macnae, 1984; Oristaglio and Hohmann, 1984). Otherwise, according to equation (4), approximating the air with a highlyresistive layer would require very small initial time steps. The boundary condition at the earth-air interface involves the calculation of the horizontal components of $\dot{b}$ in the air in order to advance the electric field at the Earth's surface. The derivation of the boundary condition is based on the validity of the vector Laplacian equation for the field in air (Oristaglio and Hohmann, 1984). This allows one to apply methods for the continuation of potential fields (Grant and West, 1965), which are also known from gravity and magnetic methods (Militzer and Weber, 1984). We carry out the upward continuation of the magnetic induction time derivative in the wavenumber domain, employing a parallel 2-D spatial Fast Fourier Transform (FFT) for efficiency.

\section{Initial conditions}

A significant advantage of our method is that we require no simplification in the earth model to initialize the fields. In contrast, Wang and Hohmann (1993) employ semi-analytical responses of a layered half-space for field initialization. To initialize the time stepping, we compute the electric field over arbitrary conductivity distributions by decomposing it into two parts. The first one, called $\mathbf{e}_{s}$, is parallel and proportional to the source distribution $\mathbf{j}_{s}$. By extrapolation of the source distribution 
onto the FD grid, one can easily obtain $\mathbf{e}_{s}$ through Ohm's law for each grid cell that is influenced by the wire source. This automatically accounts for arbitrary conductivity distributions over the mesh.

The second part is given by the DC electric field $\mathbf{e}_{D C}$ in the model. Because the DC electric field is curl free, it is determined by applying the divergence operator to the static form of equation (1b),

$$
\nabla \cdot(\sigma \nabla \Phi)=-\nabla \cdot \mathbf{j}_{s}
$$

where $\mathbf{e}_{D C}$ can be expressed as the gradient of a potential field $\Phi$. Thus, we solve a 3-D Poisson problem as common in 3-D resistivity modeling (Dey and Morrison, 1979; Scriba, 1981; Wurmstich and Morgan, 1994; Spitzer, 1995). Here, a seven-point discrete approximation to the Poisson operator is used. The numerical procedure of discretizing this operator on a nonuniform rectangular prismatic mesh is described for example by Dey and Morrison (1979). The equation represents a sparse, diagonally dominant, positive definite linear system. It can be solved easily with an efficient preconditioned conjugate gradient solver. We utilize the AZTEC parallel iterative package (Tuminaro et al., 1999) for solving the DC field equations.

The electric fields are sampled at the time instants $t_{0}, t_{1}, \ldots, t_{n-1}, t_{n}$, with $t_{0}=0$ sec and $t_{n}=t_{n-1}+\Delta t_{n-1}$. The DuFort-Frankel method requires a history of two time steps for a consistent initialization (Richtmyer and Morton, 1967). We utilize an auxiliary field given by the curl of the magnetic field, therefore no static magnetic field $\mathbf{h}_{D C}$ needs to be calculated. The sampling of the auxiliary field is shifted by $-\frac{\Delta t}{2}$ relative to the times of the electric field. Thus, the two initial fields are given by

$$
\begin{aligned}
\mathbf{e}^{0} & =\mathbf{e}_{D C}+\mathbf{e}_{s}, \\
\nabla \times \mathbf{h}^{-\frac{1}{2}} & =\sigma \mathbf{e}_{D C}+\mathbf{j}_{s} .
\end{aligned}
$$

Note that equation (6b) results from the static form of equation (1b). 


\section{Advance of the EM field}

After field initialization, the time-stepping scheme is carried out in a leap-frog fashion. It begins with computing the magnetic induction time derivative $\dot{\mathbf{b}}^{n}$ at time $t_{n}=t_{0}$ from equation (1a),

$$
\dot{\mathbf{b}}^{n}=-\nabla \times \mathbf{e}^{n}
$$

Note that the generation of a varying field originates from the change of the electric field $\mathbf{e}_{s}$ at source shutoff. Both horizontal components $\dot{b}_{x}^{n}$ and $\dot{b}_{y}^{n}$ are calculated using equation (7). In order to explicitly enforce the divergence-free condition for $\dot{\mathbf{b}}$, the vertical component $\dot{b}_{z}^{n}$ is calculated from a discretization of equation (5a) in the same manner as exemplified for b by Wang and Hohmann (1993). This routine starts from the lower mesh boundary, where $\dot{b}_{z}=0$, and is now carried out at successively higher node levels. Then the horizontal voltages $\dot{b}_{x}^{n}$ and $\dot{b}_{y}^{n}$ above the surface are calculated by upward-continuation of $\dot{b}_{z}^{n}$ at the surface. We obtain $\dot{\mathbf{h}}^{n}$ over the whole mesh from $\dot{\mathbf{b}}^{n}$, given

$$
\dot{\mathbf{b}}^{n}=\mu \dot{\mathbf{h}}^{n}
$$

and form the curl of $\dot{\mathbf{h}}^{n}$. In order to advance the electric field from $t_{n}$ to $t_{n+1}$ by equation (1b), the auxiliary quantity $\nabla \times \mathbf{h}^{n+\frac{1}{2}}$ remains to be computed for the DuFort-Frankel scheme. It is directly obtained from rearranging the central difference

$$
\nabla \times \dot{\mathbf{h}}^{n}=\frac{\nabla \times \mathbf{h}^{n+\frac{1}{2}}-\nabla \times \mathbf{h}^{n-\frac{1}{2}}}{\Delta t_{n}} .
$$

Note that at the first time step, $\nabla \times \mathbf{h}^{n-\frac{1}{2}}$ represents the initial quantity given by equation (6b).

We found that with the curl of the magnetic field derived from $\dot{\mathbf{b}}$ in this way, the divergence-free condition needs to be carried over to the curl term. Hence, we also explicitly enforce the total electric current density to be divergence-free, 


$$
\nabla \cdot(\nabla \times \mathbf{h})=\nabla \cdot \mathbf{j}=0
$$

Again, the vertical component of $\nabla \times \mathbf{h}^{n+\frac{1}{2}}$ is computed from the horizontal components, which were obtained using equation (8). However, in contrast to $\dot{\mathbf{b}}$, the computation of the vertical component of $\nabla \times \mathbf{h}^{n+\frac{1}{2}}$ starts from the top of the mesh since $\mathbf{j}$ vanishes in air and proceeds downwards at successively lower grid levels. Finally, the electric field is advanced by (Wang and Hohmann, 1993)

$$
\mathbf{e}^{n+1}=\frac{2 \tilde{\epsilon}-\sigma \Delta t_{n}}{2 \tilde{\epsilon}+\sigma \Delta t_{n}} \mathbf{e}^{n}+\frac{2 \Delta t_{n}}{2 \tilde{\epsilon}+\sigma \Delta t_{n}}\left(\nabla \times \mathbf{h}^{n+\frac{1}{2}}-\mathbf{j}_{s}^{n+\frac{1}{2}}\right)
$$

which results from discretizing equation (1b). Afterwards, the updating scheme loops back to the calculation of $\dot{\mathbf{b}}^{n+1}$ using equation (7).

\section{Parallel implementation of the time-stepping}

The realistic simulation of complex 3-D structures may require a large number of grid nodes and can quickly push the limits of a single processor computer in terms of memory needs and processor speed. To achieve acceptable computation times, our FD scheme has been designed to run on parallel computers using the Message Passing Interface (MPI) Standard (Skjellum et al., 1993). This standard addresses the architecture of distributed memory systems in an optimal way. Thus, our parallelization approach is optimized for a distributed memory architecture, for example a Beowulf-type Linux cluster.

Given the number of mesh nodes in each dimension and the number of available processors, we divide the mesh into 3-D subsets representing the different processor domains. It is important that the mesh subsets are as equal in size as possible. Otherwise, long idle times result from an unbalanced load and thus deteriorate the parallel performance. For simplicity, suppose that each processor is assigned to only one grid node in a 3-D mesh. The time-stepping scheme requires message passing across processor boundaries, which alternates with the field updating steps. The fields 
which are needed in order to complete the field update at a given node $(i, j, k)$, yet are calculated by an adjacent processor, are called "ghost" values. Figure 1 illustrates both the domain decomposition and the message passing between node $(i, j, k)$ and its 6 neighbours.

One starts with the calculation of the horizontal magnetic induction time derivatives from the curl of electric fields by equation (7). Figure 1a shows that this requires a prior communication with nodes $(i+1, j, k),(i, j+1, k)$ and $(i, j, k+1)$ in order to complete the corresponding elementary electric loops around the horizontal magnetic fields at node $(i, j, k)$. The communication prior to the divergence-free correction is illustrated in Figure 1c. The update of $\dot{b}_{z}$ requires the previously computed ghosts $\dot{b}_{x}$ and $\dot{b}_{y}$ from nodes $(i+1, j, k)$ and $(i, j+1, k)$, respectively, as well as the "lower" $\dot{b}_{z}$ from node $(i, j, k+1)$. Note that the parallel performance of the divergence-free enforcement is limited to some extent. Because of the spatial limitation of the procedure to successively higher levels, only the nodes in the same vertical layer $k$ of processors can compute $\dot{b}_{z}$ at the same time. Therefore, it is advisable not to introduce too many vertical processor boundaries in order to limit the message passing overhead.

Now, the upward continuation-procedure of the vertical voltage components at the Earth's surface follows. Its parallel implementation is explained in an extra section below. The electric field update at node $(i, j, k)$ first involves forming the curl of the magnetic induction time derivative around horizontal edges where $e_{x}$ and $e_{y}$ are sampled. Therefore, message passing according to the stencil shown in Figure 1b is carried out. The passed components of $\mathbf{b}$ belong to the ghost nodes $(i-1, j, k)$, $(i, j-1, k)$ and $(i, j, k-1)$. Then the horizontal components of the magnetic field curl, i.e. the total electric current density $\mathbf{j}$, can be computed using equation (8). Afterwards, nodes $(i-1, j, k),(i, j-1, k)$ and $(i, j, k-1)$ pass the $\mathrm{x}_{-}^{-}, \mathrm{y}^{-}$and $\mathrm{z}^{-}$ component of $\mathbf{j}$, respectively, to node $(i, j, k)$ as also illustrated by Figure 1c. This is required for the enforcement of the divergence-free condition for the current density. Again, it is carried out in a successive manner, now starting from the upper mesh 
boundary.

\section{Parallel upward-continuation procedure}

The parallel implementation of the upward-continuation procedure requires a processor topology, which is different to the one previously described. Moreover, the topology changes during the procedure since the FFT and its accompanying interpolation routines require field values along an entire grid line, in either the $\mathrm{x}$ or $\mathrm{y}$ direction, which need to be assigned to a single processor. Hence, the processor topology will change as the fields are mapped and remapped between the $\mathrm{x}$ and $\mathrm{y}$ coordinate directions when carrying out the 2-D FFT and its corresponding inverse.

Figure 2 shows the example of four processors sharing the fields of the surface layer. Each part of the figure represents a different step of the procedure. The dividing lines represent the boundaries, within which all nodes are assigned to a single processor. Prior to the 2-D FFT, a 2-D spline interpolation is needed in order to cast the original distribution of $\dot{b}_{z}$ into an equidistant FFT grid. This requires two data remapping steps. First, the original chessboard-like distribution of the field (a) among the processors has to be equi-sampled along the y-axis. The interpolated data are now distributed in a stripe-like fashion among the processors (b). After interpolation along the y-direction, the data is passed such that the interpolation can be completed along the $\mathrm{x}$-axis (c) with a corresponding remapping among the processors. Next, the FFT into the spatial wave domain is first carried out along the $\mathrm{x}$-direction $(\mathrm{d})$ and afterwards along the $\mathrm{y}$-direction (e), which also involves an additional remapping to complete the process. After calculation of the horizontal fields from the upward-continued vertical ones, the subsequent steps now proceed in reversed order. One remap is needed by the inverse FFT and two more for the interpolation of the equally spaced horizontal magnetic fields back to the original grid (f-h). 
Unfortunately, the parallelization of the procedure offers little opportunity to be accelerated due to the poor scalability of the FFT and a high message passing overhead. Since the FFT dominates the overall computation time of the upward continuation procedure, the only significant acceleration can be achieved by using the fact that the TEM field is gradually smoothed in space with increasing time. With a smooth field, the number of equidistant grid nodes and hence the computation time for the FFT can be reduced significantly. With our employed FFT algorithms optimized for a number of data points of order $2^{n}$, we choose an appropriate initial regular grid spacing such that no undersampling occurs. After a predefined time, the spacing can be doubled. Based on a series of experiments, we found that in the presence of rapidly decaying fields, the initial grid can be changed to a coarser FFT grid, after 2-3 time decades, starting from the initial time step, without affecting the accuracy of our solution.

\section{RESULTS}

To demonstrate the versatility of our numerical solution, we have simulated a variety of examples incorporating different model complexities, field configurations and measurement time ranges. Measurements of both the electric field and the magnetic induction time derivative are simulated, where the latter will be referred to as $\partial_{t} B$ for brevity. All electric field responses correspond to the electric field component which is oriented parallel to the transmitter line. Both horizontal and vertical components of $\partial_{t} B$ are simulated. The employed FD meshes are adapted in size to the different purposes. Initial time steps are set to a value of 0.1 times the right-hand side of the accuracy condition in equation (4). The FD responses are verified against solutions of different methods, including analytical, integral-equation (IE) (Newman et al., 1986) and spectral Lanczos decomposition method (SLDM) (Druskin and Knizhnerman, 1988). We used up to 12 nodes of a SUN Fire 6800 computer for all shown 
calculations.

\section{Layered half-space}

The first example is typical for the LOTEM method and simulates the vertical and horizontal response of $\partial_{t} B$, where the latter is related to the magnetic field oriented perpendicular to the transmitter. Figure 3 shows a four-layered earth model with the resistivity decreasing with depth. The fields are extracted at distances of 500-5000 m, broadside to the transmitter $(\mathrm{Tx})$, which is a $1 \mathrm{~km}$ long horizontally grounded wire. Figure 4 shows that both components of the field are in good agreement with the analytical solution. The model contains $129 \times 111 \times 61$ grid points, with the smallest grid spacing $50 \mathrm{~m}$ and the subsurface grid boundaries at $20 \mathrm{~km}$ distance from the transmitter. For this example, we could observe effects by reflections off the mesh boundaries starting at times later than $1 \mathrm{~s}$. However, such effects can be moved out to later times by further expanding the mesh boundaries. Note that the horizontal responses of $\partial_{t} B$ clearly indicate the diffusing fields by a move out in the sign reversal.

\section{Homogeneous half-space with permeable layer}

Next, we calculate the numerical solution for a layered half-space with homogeneous resistivity $(10 \Omega \cdot \mathrm{m})$ and a permeable layer at a depth of $100 \mathrm{~m}$. The relative magnetic permeability of this layer is 10 and its thickness is $100 \mathrm{~m}$. The unrealistically high magnetic permeability contrast is chosen in order to achieve a significant influence of the permeable layer. Similar to the previous example, responses are calculated broadside to the transmitter, which is a $100 \mathrm{~m}$ long electric dipole in this case. Figure 5 shows the results along the receiver profile. The results of different time stages are transformed from the vertical $\partial_{t} B$-component into early-time and late-time apparent resistivities (Petry, 1987). One notes an excellent agreement of the FD solutions with the analytical solution calculated for the permeable layer. At 
the earliest time of $0.1 \mathrm{~ms}$ (Figure $5 \mathrm{a}$ ), the signal along the profile is still in the DC state of the transient, except for the closest stations, where the decay has already started. The DC level is not influenced by the permeable layer and thus shows a response of $10 \Omega \cdot \mathrm{m}$ beyond $200 \mathrm{~m}$. For the stations at more than $400 \mathrm{~m}$ distance, the signal at $1 \mathrm{~ms}$ is characterized by a higher amplitude compared to the response arising from a homogeneous half-space. Hence, the early-time apparent resistivity is above $10 \Omega \cdot \mathrm{m}$. The late-time curve $(0.1 \mathrm{~s})$ in Figure $5 \mathrm{~b}$ indicates a convergence towards a constant apparent resistivity along the profile, slightly greater than $10 \Omega \cdot \mathrm{m}$. The analytical response of a non-permeable half-space at $0.1 \mathrm{~s}$ (dashed line) is shown to demonstrate the clear effect of the permeable layer.

\section{Conductive 3-D block in a homogeneous half-space}

The next example compares the electric field FD response over a $1 \Omega \cdot \mathrm{m}$ conductive block in a 200 times more resistive half-space with an IE solution. Figure 6 shows a plan view of the field setup and the inhomogeneity, which has a size of $100 \mathrm{~m}$ on an edge, $100 \mathrm{~m}$ below the surface. The resulting transient in Figure 7 corresponds to a receiver position as shown in Figure $6(\mathrm{Rx})$ and is transformed to late-time apparent resistivities in order to emphasize the secondary response due to the block. The fields are excited by a $100 \mathrm{~m}$ grounded wire at $150 \mathrm{~m}$ distance from the block's center. Good agreement can be observed for both solutions. They show a convergence towards a resistivity of approximately $280 \Omega \cdot \mathrm{m}$, whereas a response due to the homogeneous half-space without block (dashed line) converges towards $200 \Omega \cdot \mathrm{m}$.

Here, the relatively resistive background required an initial time step of $\Delta \mathrm{t}_{0}=$ $3 \cdot 10^{-7} \mathrm{~s}$ in order to avoid a distorting influence of the fictitious displacement current at early times. A total of 5894 time steps is required to simulate the shown time range. This amounts to a computation time of approximately 6 hours using 9 processors, compared to approximately 5 hours needed by a single processor for the IE solution. 


\section{Complex 3-D conductor at a vertical contact}

Next, synthetic data is calculated from a 3-D model similar to an example presented by Wang and Hohmann (1993), yet with a higher model complexity. The model section in Figure 8 consists of a thin $10 \Omega \cdot \mathrm{m}$ conductive layer at the surface and an underlying laterally divided resistive earth. At the contact of the $100 \Omega \cdot \mathrm{m}$ and $300 \Omega \cdot \mathrm{m}$ resistors, a $1 \Omega \cdot \mathrm{m}$ conductive 3 -D anomaly varies in steps along the profile direction. This structure, which is $400 \mathrm{~m}$ in strike length, reaches a depth of $550 \mathrm{~m}$. Figures $9 \mathrm{a}$ and $\mathrm{b}$ show the response of the electric field and the vertical $\partial_{t} B$-component, respectively, at arbitrarily chosen locations in comparison with results obtained with SLDM. In general, the solutions compare well for each receiver location, with some discrepancies at the earliest delay times of the stations which are closest to the transmitter. The largest deviation is observed in the $\partial_{t} B$-response at $\mathrm{x}=200 \mathrm{~m}$. In order to estimate which response achieves a more accurate reproduction of the exact solution, we compare with an analytical 1-D response. It is calculated from a two-layered model with the overburden according to Figure 8 and a uniform halfspace of $100 \Omega \cdot \mathrm{m}$ below $50 \mathrm{~m}$. The comparison with this analytical solution is justified, because at the earliest times the signal near the transmitter can be expected to be dominated by the overburden and the $100 \Omega \cdot \mathrm{m}$ section. The good agreement with the 1-D result indeed indicates, that a higher accuracy is achieved by the FD solution. The FD solution utilizes a mesh with $159 \times 123 \times 71$ points and a smallest grid spacing of $\Delta_{\min }=10 \mathrm{~m}$, whereas the mesh for the SLDM solution has $57 \times 64 \times 41$ points and $\Delta_{\min }=25 \mathrm{~m}$.

It may be argued that a refinement of the grid for SLDM provides for a higher accuracy at the early times. However, as outlined by Druskin and Knizhnerman (1994), the convergence of SLDM depends on the grid aspect ratio, given by its smallest and largest spacings, the conductivity contrast and frequency. In general, the grid discretization should be fine for accurate results at early times and in conductive regions, 
yet a coarser grid is required for stability at late times and in more resistive regions. We found that designing a single grid, which makes a compromise between these complementary requirements, may take place at the expense of accuracy. Therefore, the grid cannot be arbitrarily refined without slowing the convergence and thus causing stability problems at late times. We are aware that the employed grid for SLDM may not be optimal. However, the good agreement of the sign changes, caused by the conductive dyke, in particular the latest one in Figure 9b, indicates that the stability requirements for SLDM are well satisfied.

This study exemplifies the large computational effort required by the explicit time-stepping scheme in comparison with SLDM. To simulate the shown time range required a total of 3253 time steps with an initial time step of $\Delta t_{0}=10^{-7} \mathrm{~s}$. Using 12 processors of the parallel machine requires a total computation time of $30 \mathrm{~ms}$ per grid node, whereas the SLDM solution requires $3 \mathrm{~ms}$ per grid node on a single processor of the same machine.

\section{Resistive reservoir structure in a layered host}

In contrast to the previous 3-D models, this example involves a more complex resistive anomaly in a layered background. The earth model shown in Figure 10 was initially designed by Hördt et al. (2000a) from a priori well log information and represents an underground gas storage site at St. Illiers (France). At $140 \mathrm{~m}$ depth the $50 \Omega \cdot \mathrm{m}$ sedimentary overburden is interrupted by a $20 \mathrm{~m}$ thick $15 \Omega \cdot \mathrm{m}$-layer. The $10 \Omega \cdot \mathrm{m}$-basement, starting at $310 \mathrm{~m}$ depth, encompasses the dome-shaped reservoir with the upper and lower edges at $490 \mathrm{~m}$ and $602 \mathrm{~m}$ depth, respectively. It has a strike of $2 \mathrm{~km}$ and the $25 \mathrm{~m}$ thick gas-bearing layer is assumed to have a resistivity of $200 \Omega \cdot \mathrm{m}$. In a sensitivity study, the authors simulated LOTEM responses over a profile covering the reservoir structure in order to investigate if resistivity variations at the reservoir margins are detectable. With magnetic fields less sensitive to thin 
resistive structures, the study was based on electric fields in an axial configuration, i.e. the $50 \mathrm{~m}$ long transmitter points in the profile direction and the receivers record the transmitter-parallel field.

Here, we first want to compare the FD responses with the SLDM code, as it was also employed by Hördt et al. (2000a). Figure 11a shows both inline electric fields for four different positions from the transmitter, which is located over the left reservoir edge; for now the reservoir includes the white edges (Figure 10). Although the curve pairs agree qualitatively, larger quantitative differences exist. In order to estimate the degree of accuracy of both solutions, we again utilize an analytical 1-D response at the nearest receiver and assume that the influence of the reservoir is still small at this distance. The close agreement with the 1-D response, calculated from the layered model without reservoir, indicates a more accurate FD solution. With an initial time step of $\Delta t_{0}=10^{-6} \mathrm{~s}$, a total of 13389 time steps is required to simulate the shown measurement time interval.

While the meshes of both solutions have a minimum grid size of $25 \mathrm{~m}$, they differ significantly in how the grid is stretched. The grid generating the FD solution is divided into $127 \times 90 \times 83$ nodes. In contrast, the SLDM grid is much coarser at greater distances from the transmitter and thus consists of only $43 \times 43 \times 27$ nodes. In this case, the coarse structure of the SLDM grid is required in order to achieve stability over the relatively long simulation time range of more than 3 decades. Similar to the previous example, due to the issues related to the convergence of SLDM, we could not refine the mesh without significantly deteriorating the agreement at late times.

For the mentioned sensitivity study, it is important that the FD algorithm is capable of resolving the effects of small model variations. For simulating a lower amount of gas fill, Hördt et al. (2000a) changed the resistivities of both left and right reservoir edges, marked as white blocks in Figure 10, to the value of the surrounding layer. Figure 11b shows our FD responses of both original and downsized reservoir for the 
same transmitter position as before and two receiver distances. At $1400 \mathrm{~m}$ distance, no significant difference in the results occurs, since transmitter and receiver are located above the inhomogeneity in such a way that its edges hardly cause an influence on the fields. However, at $2000 \mathrm{~m}$ one observes an altered response, indicating the influence of the right reservoir margin.

\section{CONCLUSIONS}

We have presented a versatile time-stepping scheme to solve for the diffusive Maxwell equations for galvanic source configurations. The parallel code simulates electric field and magnetic field voltage responses for 3-D models where both conductivity and magnetic permeability are functions of postion. Comparisons with analytical, IE and SLDM solutions show that accurate results are obtained over arbitrarily complicated earth models. The gas reservoir example in particular demonstrates the scheme's high degree of accuracy, which is necessary to simulate response differences caused by a relatively small model variation. An advantage of our scheme, compared to the SLDM solution technique, is its robustness in the presence of large conductivity contrasts and late simulation times. The drawback is that accuracy is achieved at the expense of a high computational time demand. This problem, inherent to an explicit time-stepping method, becomes more severe with the simulation of later times. However, the parallel implementation allows solutions within reasonable computation times and thus makes the scheme applicable for the solution of inverse modeling problems (Commer, 2003; Newman and Commer, 2004).

There also exist strategies worth investigating for improving the computational efficiency of the scheme. As we put an emphasis on accuracy, the employed FD grids may be further optimized by minimizing the number of grid points while keeping an accurate spatial sampling of the diffusing fields at larger distances from the source. Alumbaugh et al. (1996) mention that a thorough study of the outer grid design 
is necessary in order to avoid slow convergence of their implicit frequency domain scheme due to large cell aspect ratios. Although not crucial for the stability of our method, an adequate grid-stretching scheme could help to optimize the FD meshes. In addition, a material averaging scheme (Moskow et al., 1999) would allow a model parameterization that is in principle independent from the underlying FD grid, thus further relaxing the meshing constraints.

\section{ACKNOWLEDGMENTS}

This work was carried out at the University of Cologne and Sandia National Laboratories (SNL), with United States Department of Energy, Office of Basic Energy Sciences funding. SNL is a multi-program laboratory operated by the Sandia Corporation, a Lockhead Martin Company, for the United States Department of Energy under contract DE-AC04-94AL85000. Additional support for Michael Commer was provided by a short-term scholarship granted by the German Academic Exchange Service (DAAD), for G. A. Newman through a Mercator Fellowship granted by the Deutsche Forschungsgemeinschaft (DFG). We express our gratitude to Tilman Hanstein for providing the analytical results for the permeable layered model and to Vladimir Druskin and Leonid Knizhnerman for their permission to use their SLDM code. We also wish to acknowledge Steve Plimpton for providing external parallel remapping routines.

\section{REFERENCES}

Adhidjaja, J. I., and Hohmann, G. H., 1989, A finite-difference algorithm for the transient electromagnetic response of a three-dimensional body: Geophys. J. Int., 98, 233-242.

Alumbaugh, D. L., Newman, G. A., Prevost, L., and Shadid, J. N., 1996, Three- 
dimensional wideband electromagnetic modeling on massively parallel computers: Radio Science, 31, 1-23.

Ascher, U. M., and Petzold, L., 1998, Computer methods for ordinary differential equations and differential-algebraic equations: SIAM, Philadelphia.

Commer, M., 2003, Three-dimensional inversion of transient electromagnetic data: A comparative study: Ph.D. thesis, Universität zu Köln.

Dey, A., and Morrison, H. F., 1979, Resistivity modeling for arbitrarily shaped threedimensional structures: Geophysics, 44, 753-780.

Druskin, V. L., and Knizhnerman, L. A., 1988, A spectral semi-discrete method for the numerical solution of 3D-nonstationary problems in electrical prospecting: Physics of the solid Earth, 24, 641-648.

Druskin, V., and Knizhnerman, L., 1994, Spectral approach to solving threedimensional Maxwell's diffusion equations in the time and frequency domains: Radio Science, 29, 937-953.

DuFort, E. C., and Frankel, S. P., 1953, Stability conditions in the numerical treatment of parabolic differential equations: Math. tables and other aids to comput. (former title of Math. Comput.), 7, 135-152.

Edwards, R. N., and Nabighian, M. N., 1991, The Magnetometric Resistivity Method in Nabighian, M. N., Ed., Electromagentic Methods in Applied Geophysics: Soc. Expl. Geophys., 47-104.

Goldman, M. M., and Stoyer, C. H., 1983, Finite-difference calculations of the transient field of an axially symmetric earth for vertical magnetic dipole excitation: Geophysics, 48, 953-963. 
Grant, F., and West, G. F., 1965, Interpretation theory in applied gepophysics: McGraw-Hill, New York.

Haber, E., Ascher, U. M., and Oldenburg, D., 2002, 3d forward modelling of timedomain electromagnetic data: Expanded Abstracts, 641-644, Soc. Expl. Geophys., 72nd Annual Meeting, Salt Lake City, Utah.

Haber, E., 2000, A mixed finite element method for the solution of the magnetostatic problem in 3d: Computational Geosciences, 4, 323-326.

Hördt, A., Druskin, V. L., and Knizhnerman, L. A., 1992, Interpretation of 3-D effects on long-offset transient electromagnetic (LOTEM) soundings in the Münsterland area/Germany: Geophysics, 57, 1127-1137.

Hördt, A., Andrieux, P., Neubauer, F. M., Rüter, H., and Vozoff, K., 2000a, A first attempt at monitoring underground gas storage by means of time-lapse multichannel transient electromagnetics: Geophys. Prospect., 48, 489-509.

_ 2000b, Interpretation of long-offset transient electromagnetic data from the Odenwald area, Germany, using two-dimensional modelling: Geophys. J. Int., 140, $577-586$.

Lapidus, L., and Pinder, G. F., 1982, Numerical solution of partial differential equations in science and engineering: J. Wiley and Sons, New York.

Macnae, J. C., 1984, Survey design for multicomponent electromagnetic systems: Geophysics, 49, 265-273.

Militzer, H., and Weber, F., 1984, Angewandte Geophysik: Springer, Heidelberg.

Mitsuhata, Y., Uchida, T., and Amano, H., 2002, 2.5-D inversion of frequency-domain electromagnetic data generated by a grounded-wire source: Geophysics, 67, 17531768. 
Moskow, S., Druskin, V., Habashy, T., Lee, P., and Davydychewa, S., 1999, A finite difference scheme for elliptic equations with rough coefficients using a cartesian grid nonconforming to interfaces: SIAM J. Numerical Analysis, 36, 442-464.

Newman, G., and Alumbaugh, D. L., 1997, Three-dimensional massively parallel electromagnetic inversion - I. Theory: Geophys. J. Int., 128, 345-354.

Newman, G. A., and Commer, M., 2004, Three dimensional transient electromagnetic inversion: Problem formulation and method of solution: submitted to Geophys. J. Int.

Newman, G. A., Hohmann, G. W., and Anderson, W. L., 1986, Transient electromagnetic response of a three-dimensional body in a layered earth: Geophysics, $\mathbf{5 1}$, $1608-1627$.

Oristaglio, M. L., and Hohmann, G. W., 1984, Diffusion of electromagnetic fields into a two-dimensional earth: A finite-difference approach: Geophysics, 49, 870-894.

Petry, H., 1987, Transient elektromagnetische Tiefensondierungen — Modellrechnungen und Inversion: Master's thesis, Univ. zu Köln, Inst. für Geophys. und Meteo.

Richtmyer, R. D., and Morton, K. W., 1967, Difference methods for initial value problems: Interscience publishers, New York.

Scriba, H., 1981, Computation of the electric potential in three-dimensional structures: Geophys. Prospect., 29, 790-802.

Skjellum, A., Doss, N. E., and Bangalore, P. V., 1993, Writing libraries in MPI, in Skjellum, A., and Reese, D., Eds., Proceedings of the Scalable Parallel Libraries Conference: IEEE Comput. Sci. Press, 166-173.

Spies, B. R., and Frischknecht, F. C., 1991, Electromagnetic Sounding, in Nabighian, M. N., Ed., Electromagentic Methods in Applied Geophysics: Soc. Expl. Geophys. 
Spitzer, K., 1995, A 3-D finite-difference algorithm for DC resistivity models using conjugate gradient methods: Geophys. J. Int., 123, 903-914.

Strack, K. M., 1992, Exploration with Deep Transient Electromagnetics: Elsevier, Amsterdam.

Thern, H., Hanstein, T., and Hördt, A., 1996, LOTEM im Odenwald - auch hier ein Krustenleiter?, in Bahr, K., and Junge, A., Eds., Protokoll über das 16. Kolloquium Elektromagnetische Tiefenforschung: Deuts. Geophys. Gesellschaft, 341-350.

Tuminaro, R. S., Heroux, M., Hutchinson, S. A., and Shadid, J. N., 1999, Official AZTEC Users Guide Version 2.1, Sandia National Laboratories Report SAND998801J.

Wang, T., and Hohmann, G. W., 1993, A finite-difference, time-domain solution for three-dimensional electromagnetic modeling: Geophysics, 58, 797-809.

Wurmstich, B., and Morgan, F. D., 1994, Modeling of streaming potential responses caused by oil well pumping: Geophysics, 59, 46-56.

Yee, K. S., 1966, Numerical solution of initial boundary problems involving Maxwell's equations in isotropic media: IEEE Trans. Ant. Prop., AP-14, 302-309. 


\section{FIGURES}

FIG. 1. The 3-D staggered grid as used for discretizing Maxwell's equations. (a) Electric fields are sampled on cell edges, (b) magnetic fields are sampled on cell faces. The figure also illustrates the communication scheme for the parallel field update. Node $(i, j, k)$ has assigned to it electric fields $e_{x}\left(i+\frac{1}{2}, j, k\right), e_{y}\left(i, j+\frac{1}{2}, k\right)$ and $e_{z}\left(i, j, k+\frac{1}{2}\right)$ and magnetic fields $h_{x}\left(i, j+\frac{1}{2}, k+\frac{1}{2}\right), h_{y}\left(i+\frac{1}{2}, j, k+\frac{1}{2}\right)$ and $h_{z}\left(i+\frac{1}{2}, j+\frac{1}{2}, k\right)$, including their corresponding time derivatives. See text for additional details regarding the parallel field update.

FIG. 2. Parallel field upward continuation scheme involving remapping, interpolation and both forward and inverse 2-D FFT along both horizontal dimensions $\mathrm{x}$ and $\mathrm{y}$ of the surface grid. (a) Initial distribution, (b) remap, y-interpolation, (c) remap, x-interpolation, (d) FFT(x), (e) remap, FFT(y), upward continuation, $\mathrm{FFT}^{-1}(\mathrm{y})$, remap, $\mathrm{FFT}^{-1}(\mathrm{x})$, (f) x-interpolation, (g) remap, y-interpolation, (h) remap to initial distribution.

FIG. 3. Layered model for comparing the FD response with an analytical solution. The receiver profile is oriented perpendicular to the transmitter direction.

FIG. 4. (a) Vertical and (b) horizontal FD response of $\partial_{t} B$ in comparison with analytical (dashed line) solutions for the layered model shown in Figure 3.

FIG. 5. Comparison of the (a) early-time and (b) late-time apparent resistivity calculated from the vertical component of $\partial_{t} B$ at the surface of a homogeneous half-space with a permeable layer. The FD solution is compared with an analytical layered solution.

FIG. 6. Plan view of the 3-D model for comparing with an IE solution. The conduc- 
tive block is buried $100 \mathrm{~m}$ below the surface.

FIG. 7. Late-time apparent resistivities calculated from the electric field due to the model in Figure 6. The dashed line corresponds to an analytical half-space response without inhomogeneity.

FIG. 8. Section view of a complex 3-D anomaly at a vertical contact. The transmitter direction is parallel to the strike of the conductor.

FIG. 9. Response of the (a) electric field and (b) the vertical component of $\partial_{t} B$ in comparison with the SLDM (dashed line) solution for different receiver positions over the 3-D model shown in Figure 8.

FIG. 10. Section through the 3-D model approximating the St. Illiers underground gas storage site (Hördt et al., 2000a). The reservoir extends $2 \mathrm{~km}$ in the direction perpendicular to the drawing plane without variation.

FIG. 11. (a) Comparison of the FD (solid line) and SLDM (dashed line) solution for four receiver points over the St. Illiers-model shown in Figure 10. The shown 1-D solution corresponds to the same model without reservoir. (b) Comparison of FD solutions at two receiver positions for different resistivities of the reservoir edge. 
a)

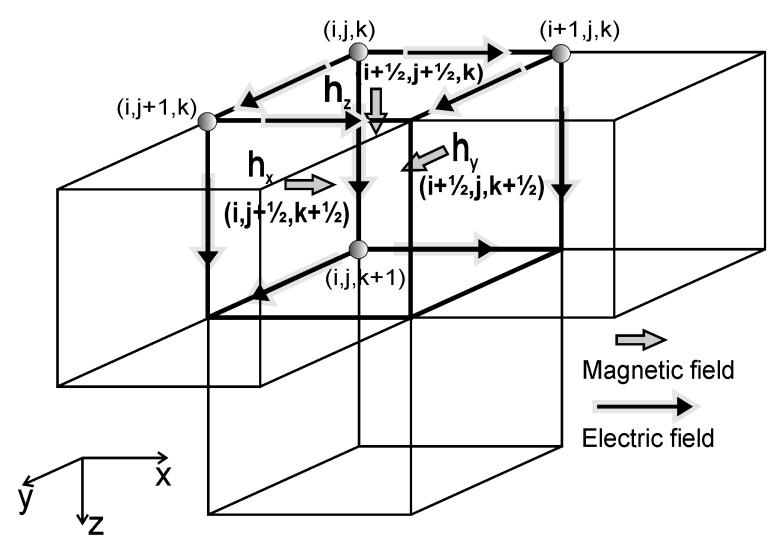

b)

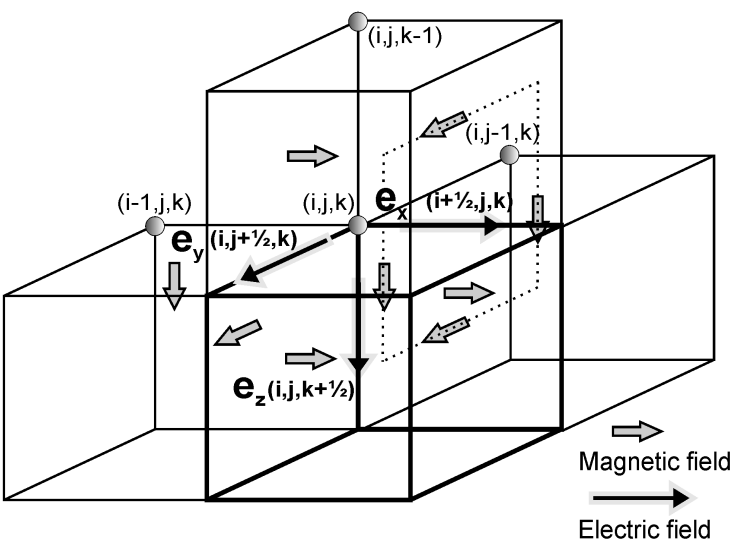

c)

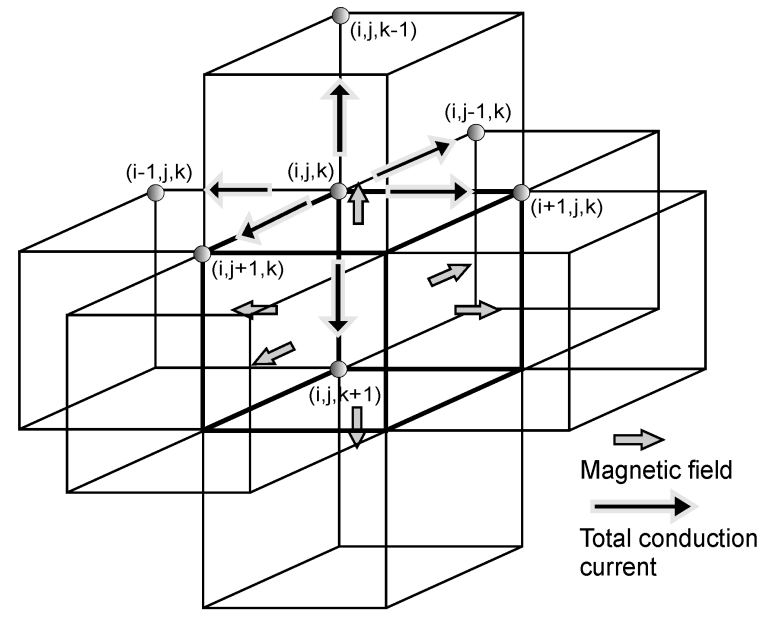

FIG. 1. The 3-D staggered grid as used for discretizing Maxwell's equations. (a) Electric fields are sampled on cell edges, (b) magnetic fields are sampled on cell faces. The figure also illustrates the communication scheme for the parallel field update. Node $(i, j, k)$ has assigned to it electric fields $e_{x}\left(i+\frac{1}{2}, j, k\right), e_{y}\left(i, j+\frac{1}{2}, k\right)$ and $e_{z}\left(i, j, k+\frac{1}{2}\right)$ and magnetic fields $h_{x}\left(i, j+\frac{1}{2}, k+\frac{1}{2}\right), h_{y}\left(i+\frac{1}{2}, j, k+\frac{1}{2}\right)$ and $h_{z}\left(i+\frac{1}{2}, j+\frac{1}{2}, k\right)$, including their corresponding time derivatives. See text for additional details regarding the parallel field update. 
a)

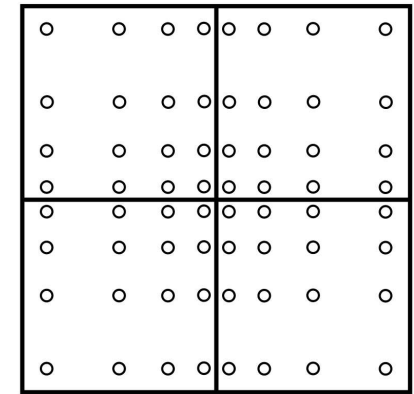

b)

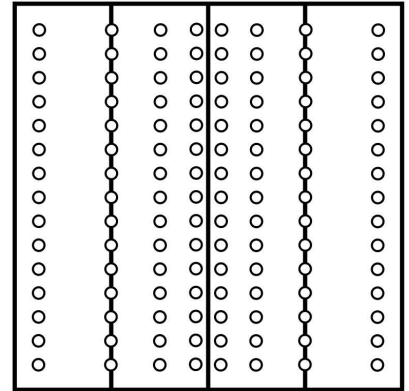

C)

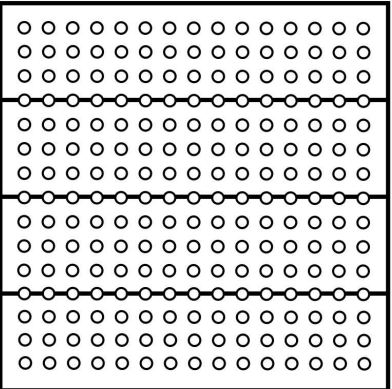

d)

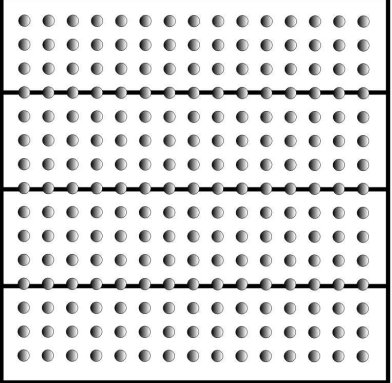

e)

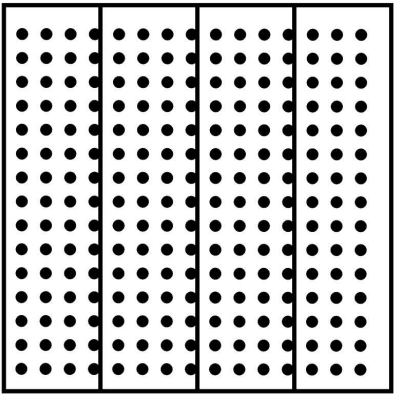

f)

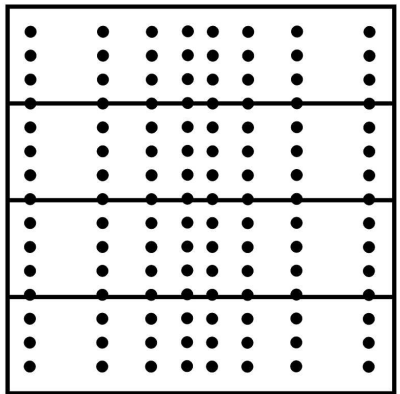

g)

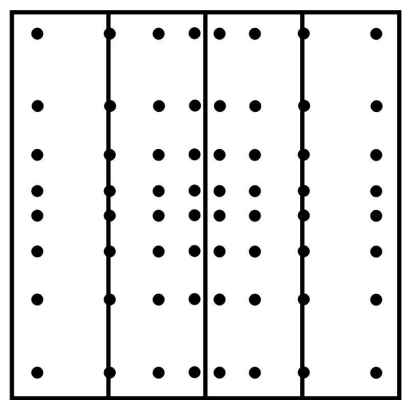

h)

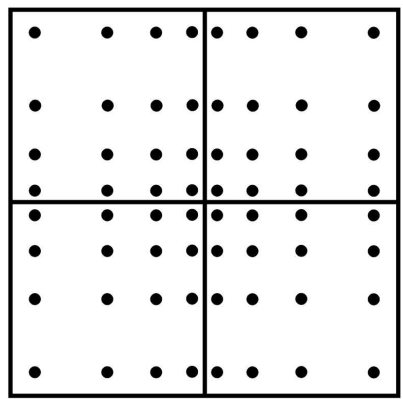

FIG. 2. Parallel field upward continuation scheme involving remapping, interpolation and both forward and inverse 2-D FFT along both horizontal dimensions $\mathrm{x}$ and $\mathrm{y}$ of the surface grid. (a) Initial distribution, (b) remap, $\mathrm{y}$-interpolation, (c) remap, $\mathrm{x}$-interpolation, (d) $\mathrm{FFT}(\mathrm{x}),(\mathrm{e}) \operatorname{remap}, \mathrm{FFT}(\mathrm{y})$, upward continuation, $\mathrm{FFT}^{-1}(\mathrm{y}), \operatorname{remap}, \mathrm{FFT}^{-1}(\mathrm{x})$, (f) $\mathrm{x}$-interpolation, (g) remap, y-interpolation, (h) remap to initial distribution. 


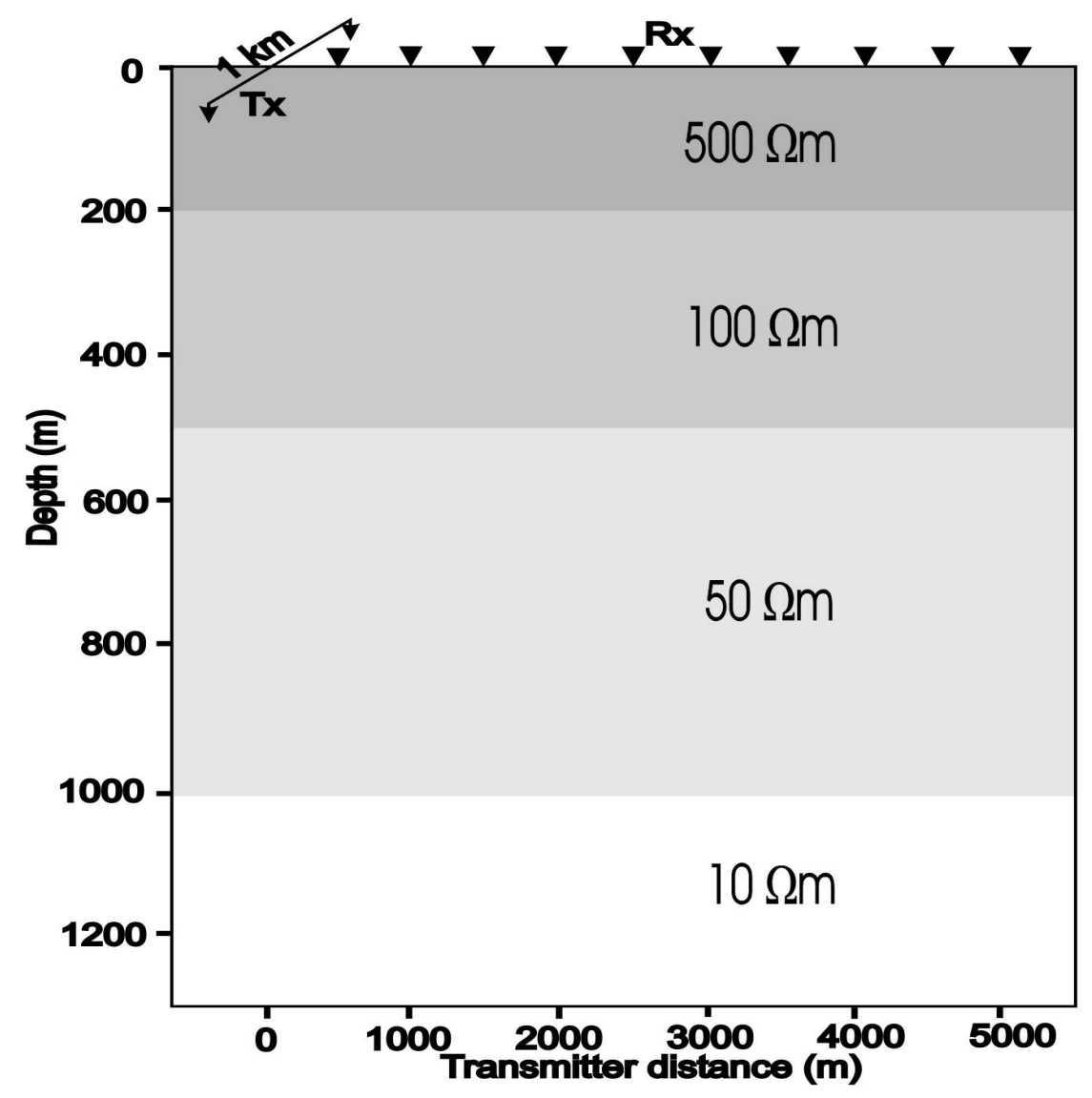

FIG. 3. Layered model for comparing the FD response with an analytical solution. The receiver profile is oriented perpendicular to the transmitter direction. 

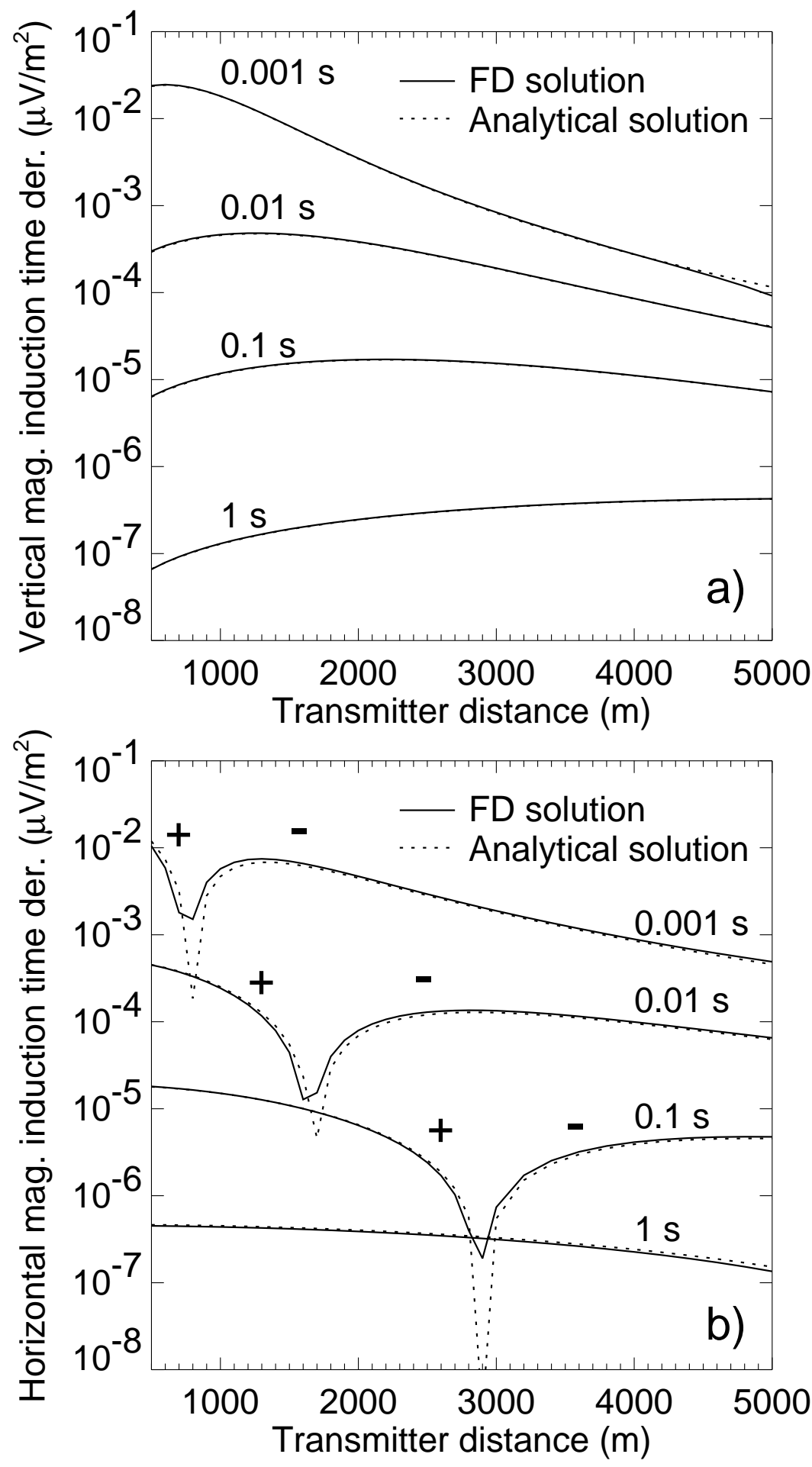

FIG. 4. (a) Vertical and (b) horizontal FD response of $\partial_{t} B$ in comparison with analytical (dashed line) solutions for the layered model shown in Figure 3. 

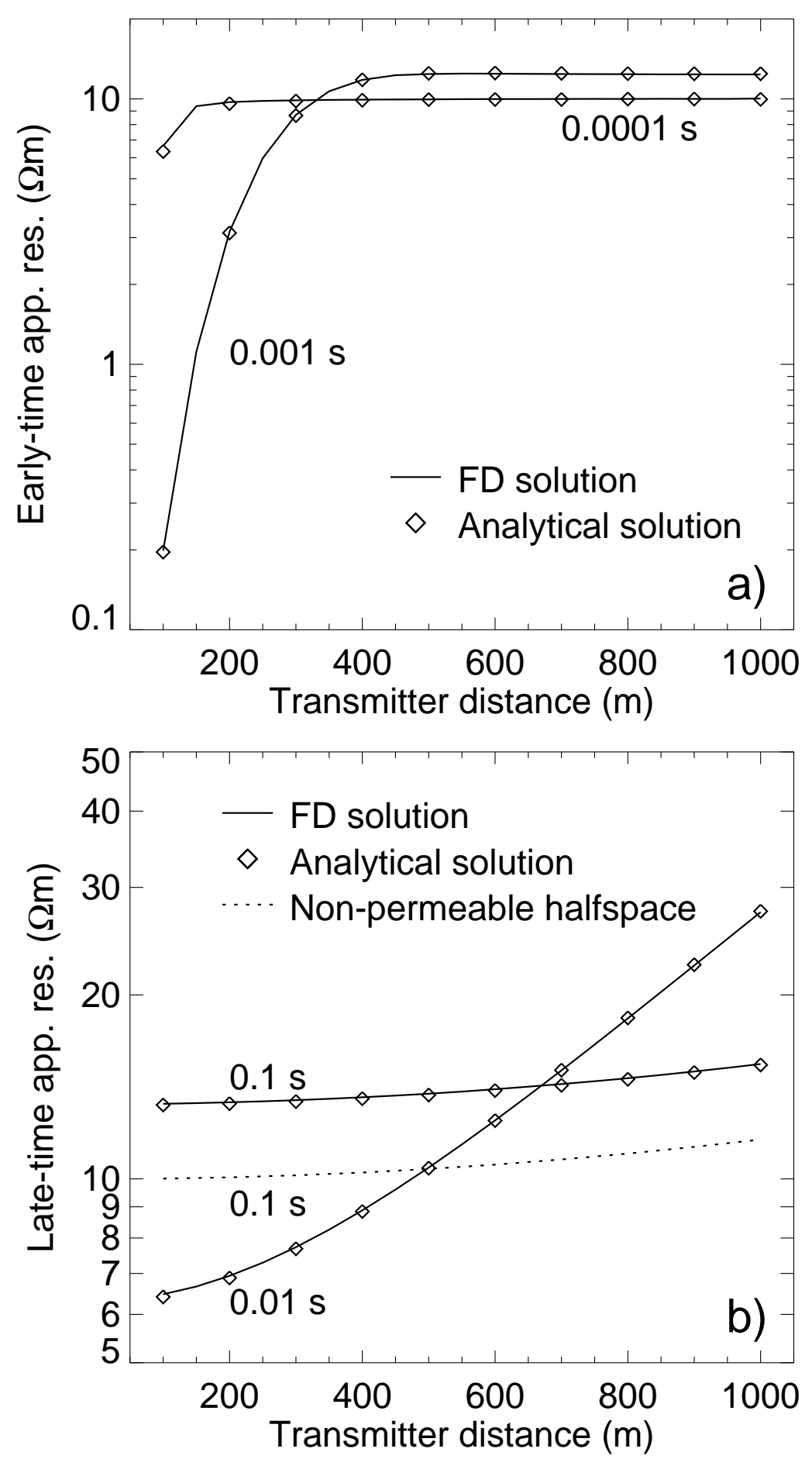

FIG. 5. Comparison of the (a) early-time and (b) late-time apparent resistivity calculated from the vertical component of $\partial_{t} B$ at the surface of a homogeneous half-space with a permeable layer. The FD solution is compared with an analytical layered solution. 


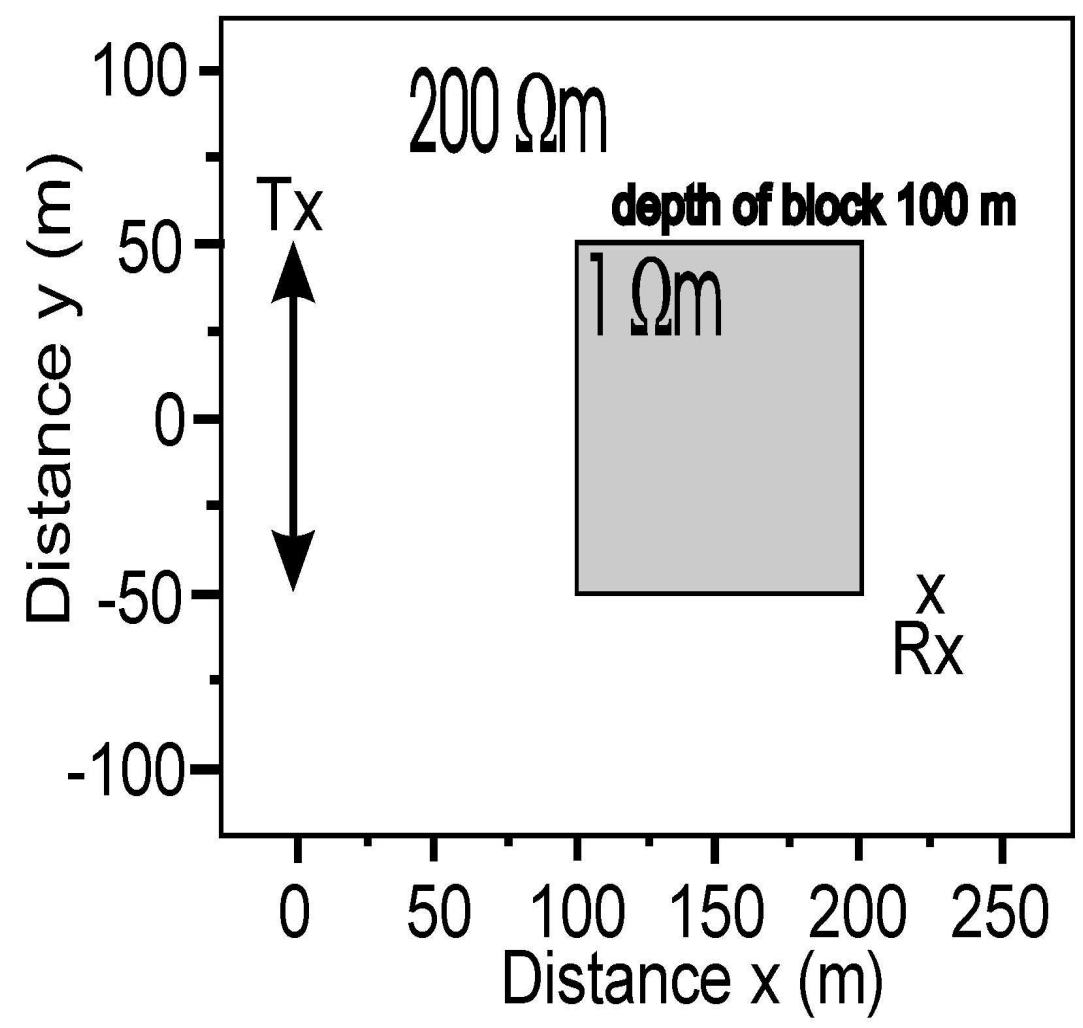

FIG. 6. Plan view of the 3-D model for comparing with an IE solution. The conductive block is buried $100 \mathrm{~m}$ below the surface. 


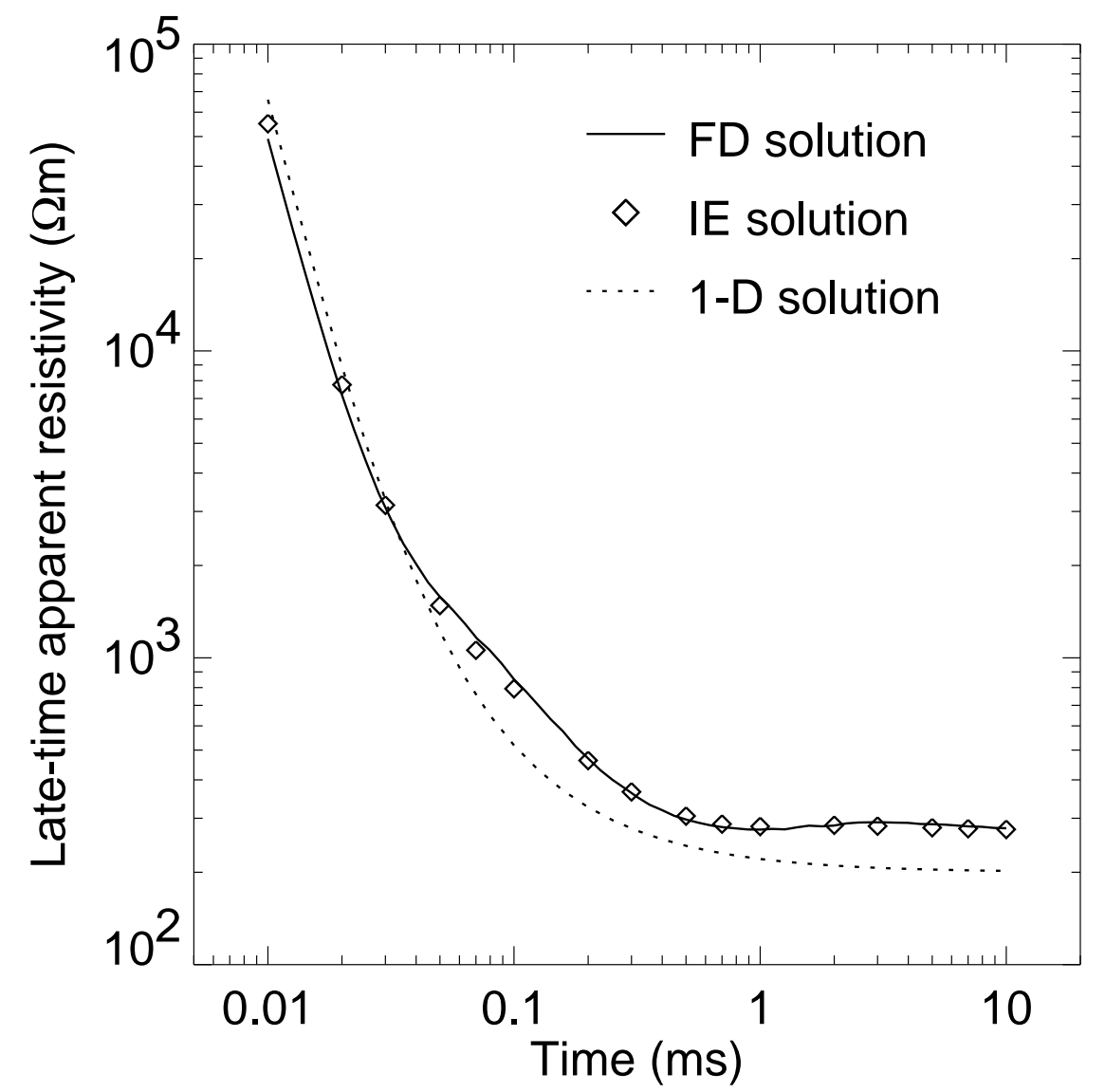

FIG. 7. Late-time apparent resistivities calculated from the electric field due to the model in Figure 6. The dashed line corresponds to an analytical half-space response without inhomogeneity. 


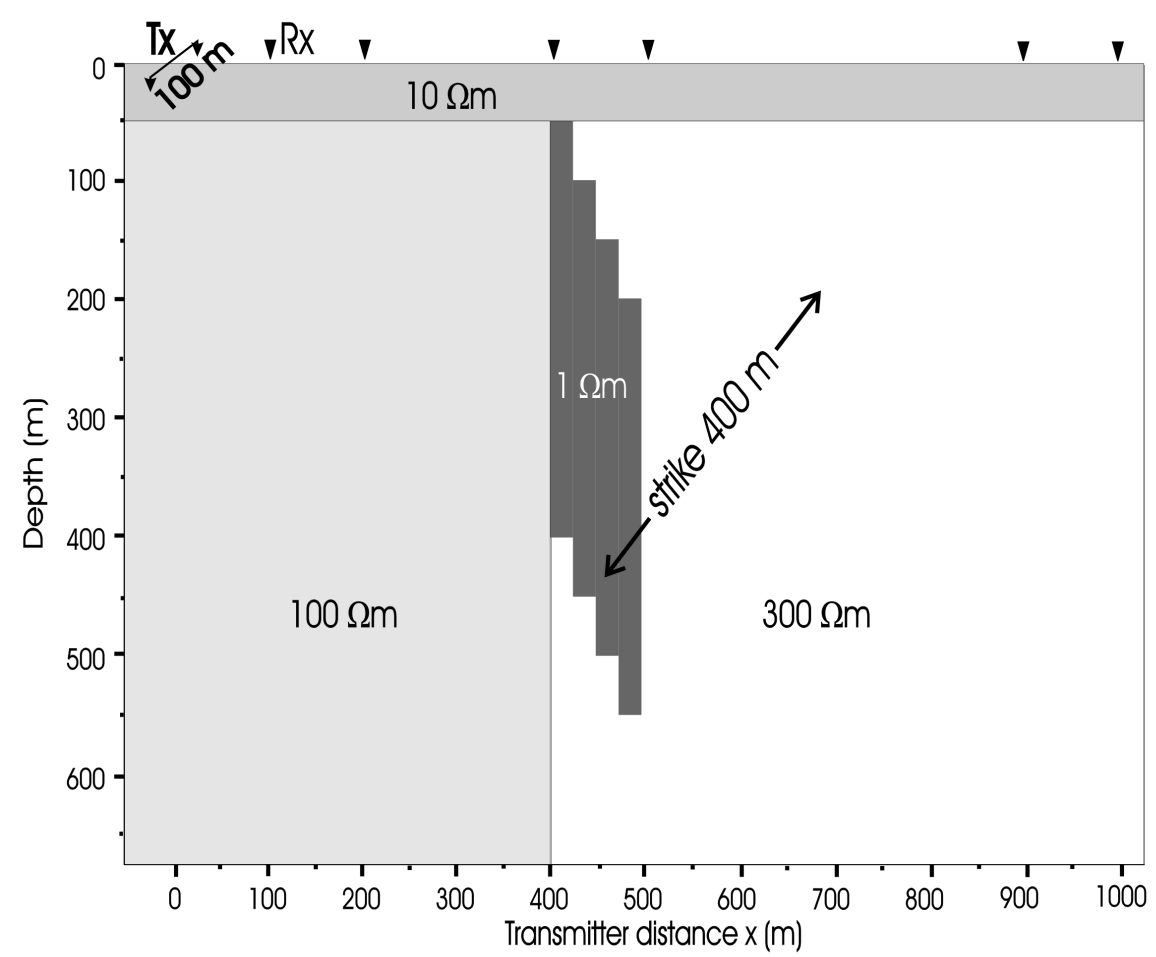

FIG. 8. Section view of a complex 3-D anomaly at a vertical contact. The transmitter direction is parallel to the strike of the conductor. 

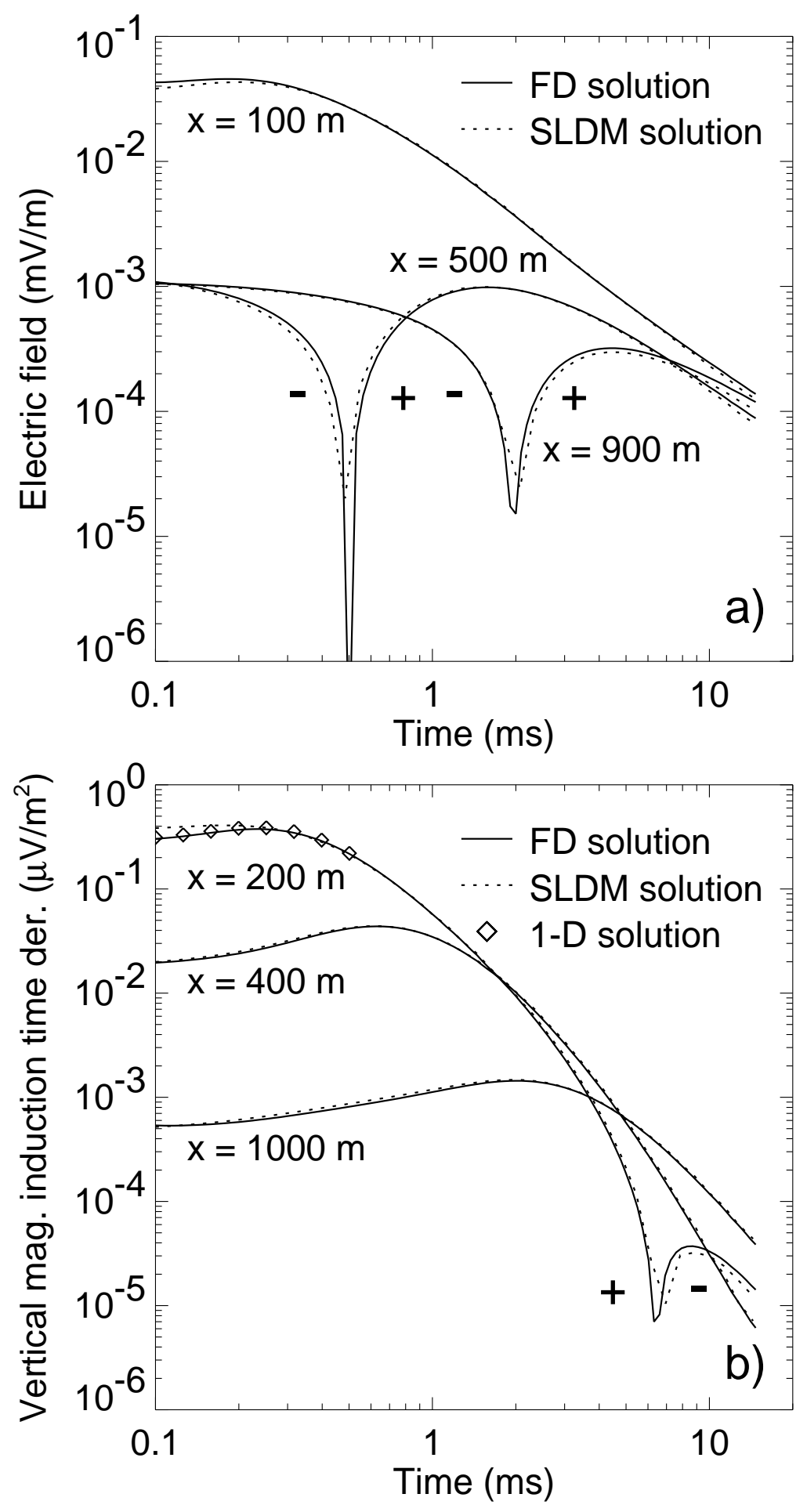

FIG. 9. Response of the (a) electric field and (b) the vertical component of $\partial_{t} B$ in comparison with the SLDM (dashed line) solution for different receiver positions over the 3-D model shown in Figure 8. 


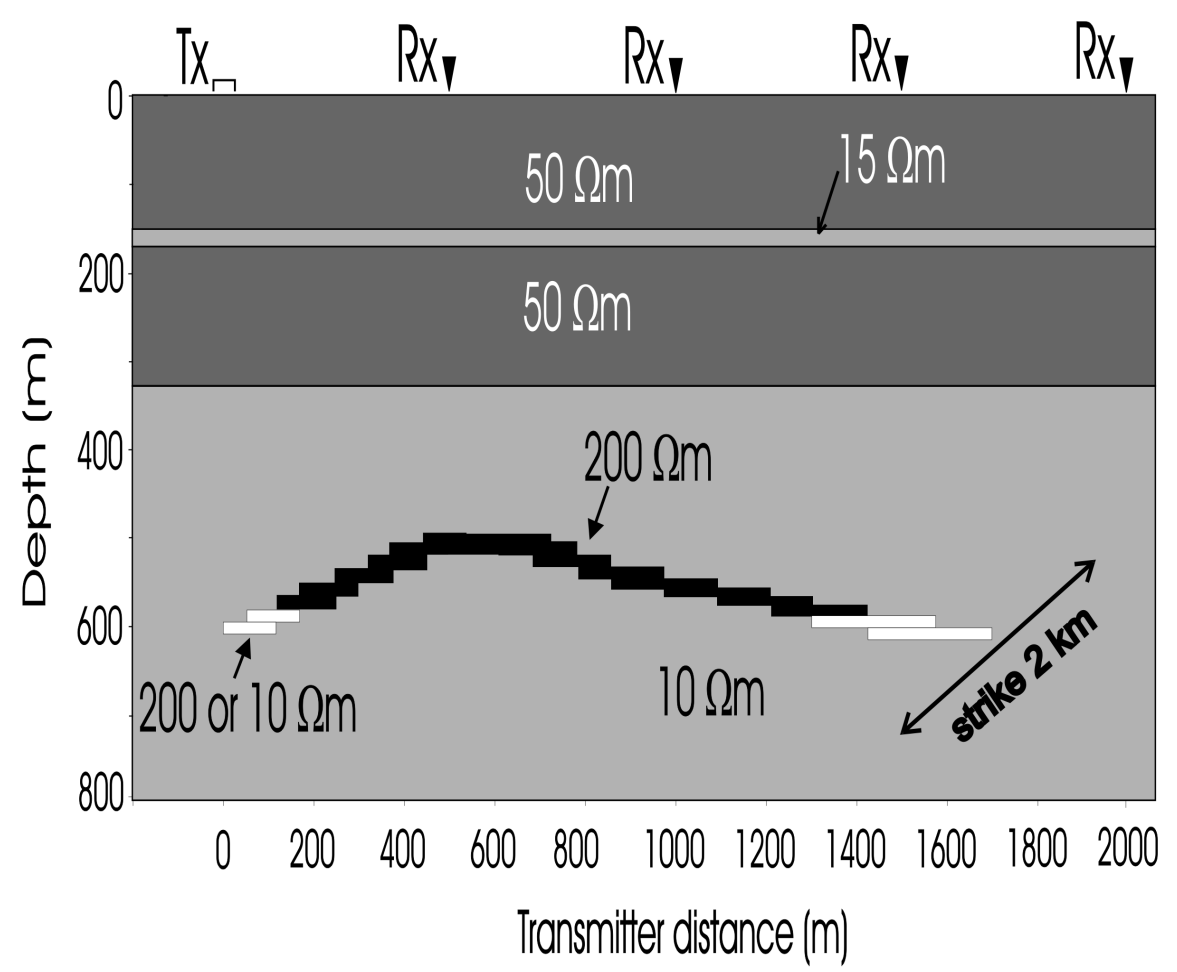

FIG. 10. Section through the 3-D model approximating the St. Illiers underground gas storage site (Hördt et al., 2000a). The reservoir extends $2 \mathrm{~km}$ in the direction perpendicular to the drawing plane without variation. 

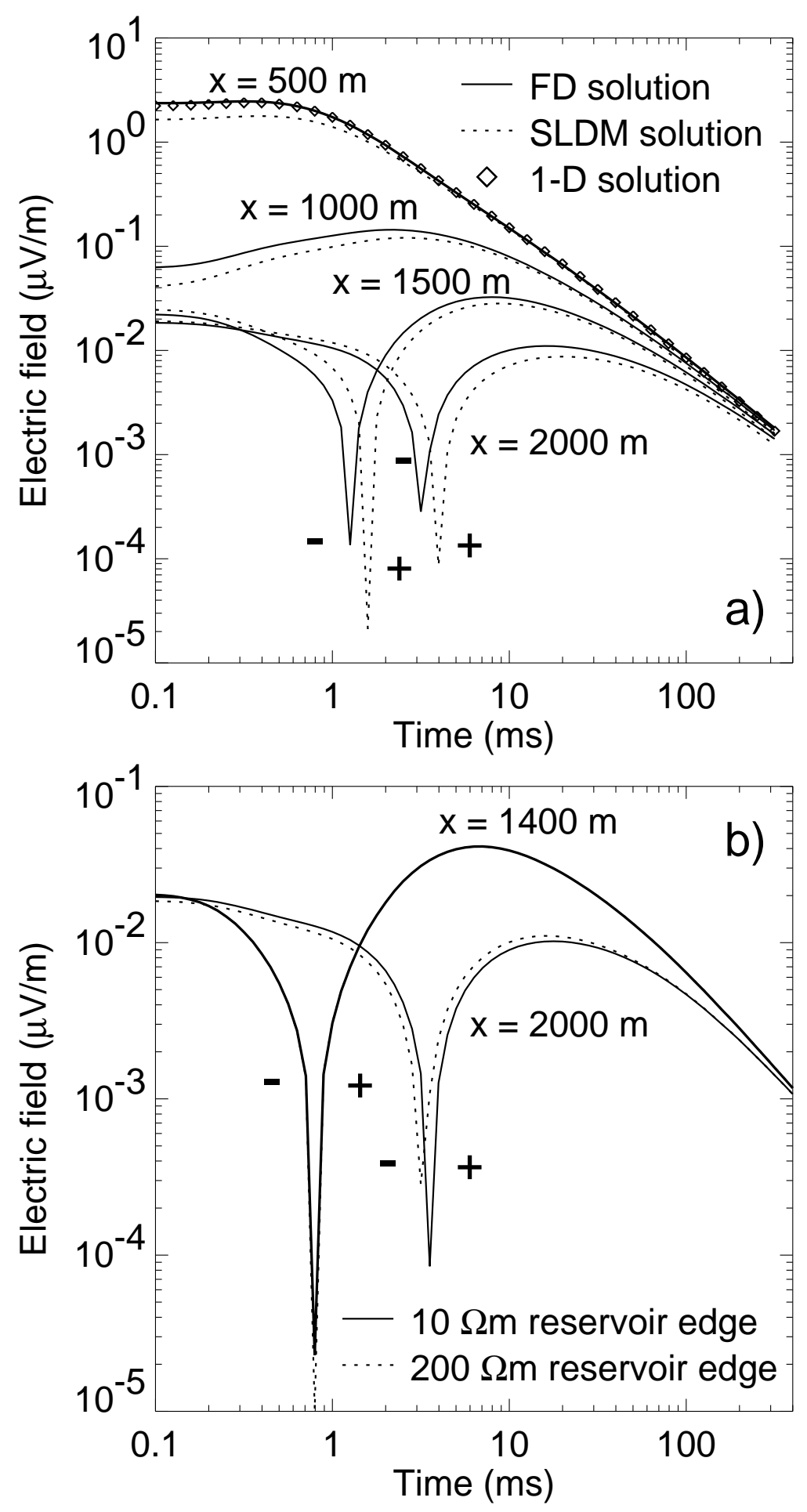

FIG. 11. (a) Comparison of the FD (solid line) and SLDM (dashed line) solution for four receiver points over the St. Illiers-model shown in Figure 10. The shown 1-D solution corresponds to the same model without reservoir. (b) Comparison of FD solutions at two receiver positions for different resistivities of the reservoir edge. 